\title{
Splenic infarction associated with acute infectious mononucleosis
}

Yuji Suzuki, Keisuke Kakisaka, Hidekatsu Kuroda, Tokio Sasaki, and Yasuhiro Takikawa

Division of Hepatology, Department of Internal Medicine, Iwate Medical University School of Medicine, Morioka, Japan
Received: November 29, 2016 Revised : December 19, 2016 Accepted: December 19, 2016

\author{
Correspondence to \\ Yuji Suzuki, M.D. \\ Tel: +81-19-651-5111 \\ Fax: +81-19-652-6664 \\ E-mail:yusuzuki@iwate-med.ac.jp
}

A 22-year-old man was admitted to our hospital with a 3-day history of left upper quadrant abdominal pain. Five days prior to admission, he had continuous fatigue, fever, and sore throat lasting for 1 week. Laboratory studies on admission showed a white blood cell count of $6,240 / \mu \mathrm{L}$ (normal range, 3,210 to 9,680 ) with $51 \%$ lymphocytes, $8 \%$ of which were atypical. A contrast-enhanced ultrasound sonography using Sonazoid (perfluorobutane; Daiichi Sankyo, Tokyo, Japan) revealed an enlarged spleen with hypoechoic wedgeshaped areas with absence of blood flow (Fig. $1 \mathrm{~A}$ and $1 \mathrm{~B}$ ). A computed tomography (CT) scan of the abdomen revealed splenic enlargement with splenic in-
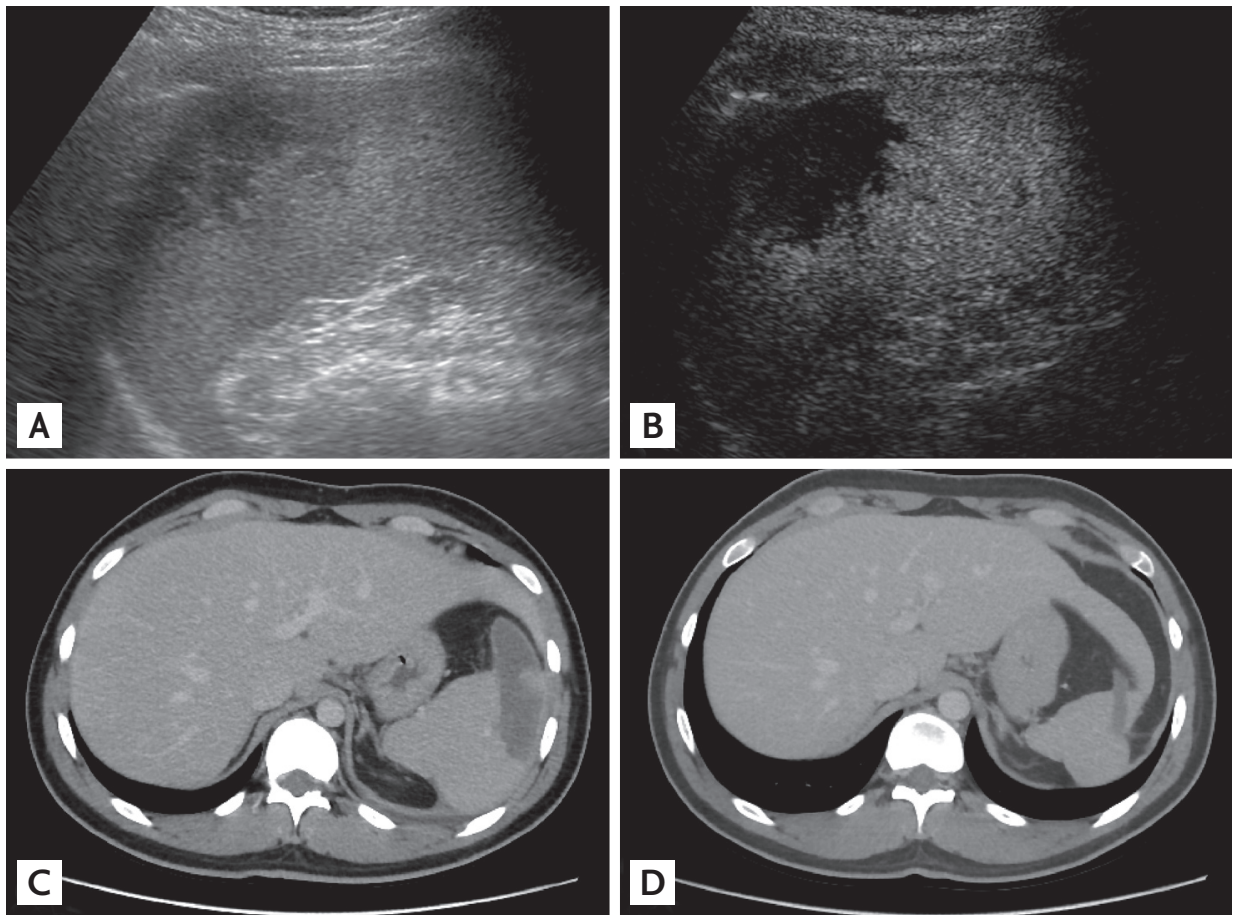

Figure 1. Abdominal imaging in the patient. (A) Ultrasound sonography showed a peripheral wedge-shaped hypoechoic area in an enlarged spleen. (B) Late-vascular phase (i.e., 6o seconds after injection) of contrast-enhanced ultrasound sonography with Sonazoid(Daiichi Sankyo, $0.015 \mathrm{~mL} / \mathrm{kg}$ ) showed a non-enhanced infarcted area as a wedge-shaped area. (C) A portal venous phase computed tomography (CT) taken on the admission day showed splenomegaly and a wedge-shaped hypoenhancement area. (D) A portal venous phase CT after 2 months of discharge revealed decreased splenic infarcted areas accompanied by localized atrophic changes in the infarcted areas. 
farcts (Fig. 1C). Acute Epstein-Barr virus (EBV) infection was confirmed by assessing serum EBV-DNA using polymerase chain reaction. Serum EBV-DNA was positive at the time of admission as well as 10 days later. From these observations, we concluded that the patient had infectious mononucleosis (IM) due to EBV infection with splenic infarction. Tests for thrombophilia were normal. After 16 days of conservative treatment, the patient was discharged without any complications. A repeat CT scan 2 months after discharge showed reduction of the infarct area accompanied by atrophic changes (Fig. 1D).

IM is characterized by a triad of fever, pharyngitis, and lymphadenopathy. Splenic infarction is a rare but possible complication of IM. The precise etiology of splenic infarction associated with IM remains unknown. Abdominal pain is the most common complaint associated with splenic infarction, and the pain is most often located in the left upper quadrant. Although splenic infarction is typically managed conservatively, splenic rupture-which is a life-threatening complication of IM-may require splenectomy. Taken together, careful investigation using ultrasound sonography or CT should be considered for accurate diagnosis of IM patients with abdominal pain.

\section{Conflict of interest}

No potential conflict of interest relevant to this article was reported. 